\title{
Recursive Configuration with Common Ground Tracks of LEO Satellite Constellations for Area Coverage
}

\author{
Shaofei Meng ${ }^{a)}$, Qi Yang ${ }^{b}$, Jian Wu ${ }^{\text {c) }}$, Qingguo Liu ${ }^{d)}$ \\ Xi'an Research Institute of High-tech, Xi'an 710025, China \\ a) shaofei_m@sina.com \\ b)epgc_yangqi@163.com \\ c)wujian6029@163.com \\ d)teamalpha@163.com
}

Keywords: LEO Satellite, Tracks, Area Coverage

\begin{abstract}
With the widely use of modern evolutionary algorithm in constellation configuration design for area coverage, the selection of basic configuration before the optimization is becoming more and more important. To design a good configuration suitable for area coverage, the orbit selection criterions are put forward. Then the iterative algorithm for recursive orbit parameters is researched. In addition, the advantages of recursive orbit and configuration with common ground tracks are combined and the new configuration is put forward and analyzed. To verify the better coverage performance, the coverage performance of Walker type and the new configuration are simulated and compared. The result suggests that the new configuration have better coverage performance and more suitable for area coverage.
\end{abstract}

\section{Introduction}

Satellite constellation is important area of modern aerospace, and constellation configuration design is one of the key technologies restricting the development of satellite constellation. In tradition, the constellation is analytically designed mainly for continuous global coverage using spherical geometry knowledge, leading to the well-known Star type and Walker types [1, 2], etc. With the widely use of computer in aerospace area, constellation for zonal or area coverage is greatly developed based on modern evolutionary algorithms [3 5], such as genetic algorithm [6], NSGA-II algorithm [7] and SPE2 algorithm, etc.

However, as there are six elements for one satellite and dozens even hundreds of satellites for one constellation, the optimization variables space is always too large. So the basic configurations are always determined at the beginning of optimization so that the satellites have some common parameters, usually major semi-axis, electricity and inclination, and the evolutionary algorithm is only used in the other parameters optimization [8 10]. The determination of the basic configuration really decreases the calculation quantity and increases the optimization speed, but restricts the coverage performance of the optimal constellation either. So it's important to find out an optimal basic configuration for area coverage.

\section{Orbit Selection Criterion}

Satellite coverage performance is mainly includes the length of coverage time and the range of coverage area. As the LEO satellites always have small electricity, their coverage time is nearly evenly distributes in the orbit. So for the LEO satellites, their coverage performances are always differ in range of coverage area.

Assume the satellite position in time space represents the satellite coverage resource, and then the ground tracks represent the distribution of coverage resource. It's also assumed that the 
coverage resource is usable where the area is covered. Then following criterions are put forward:

(1)The ground tracks of different periods are more integrated, the rate of usable coverage resource is higher. That is because for limited area, if the ground tracks are more closed, the maximum length of the tracks in the area could be greater. As an extreme example shown in Fig.1, for the geostationary satellite, it the ground tracks are all integrated in the area, the rate of usable coverage resource will be $100 \%$.

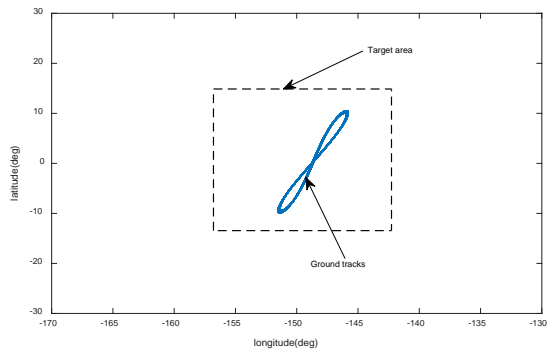

FIGURE.1 Maximum rate of usable coverage resource

(2)The whole length of all the tracks are shorter, the rate of usable coverage resource is higher. That is because if the whole length of all the tracks is shorter, the rate of the length of the tracks in the area to the whole length could be larger by adjusting the satellite orbit parameters.

For example, when satellites in recursive orbit, its ground tracks will repeat after a regression cycle. The length of ground tracks in target area is shorter than that of satellites in nonrecurrent orbit. Take values of their orbit parameters as $a=8672.1 \mathrm{~km}, e=5.75 e-02, i=76.81^{\circ}$ (for recursive orbit) and $i=103.19^{\circ}$ (for nonrecurrent orbit), $\Omega=\omega=\theta_{0}=0^{\circ}$, and simulate time $T=12 d$. The target area and the ground tracks are shown in Fig.2. The target area is divided into small units and the mean value of their coverage time is calculated to be 9961s for recursive orbit and 9880s for nonrecurrent orbit.

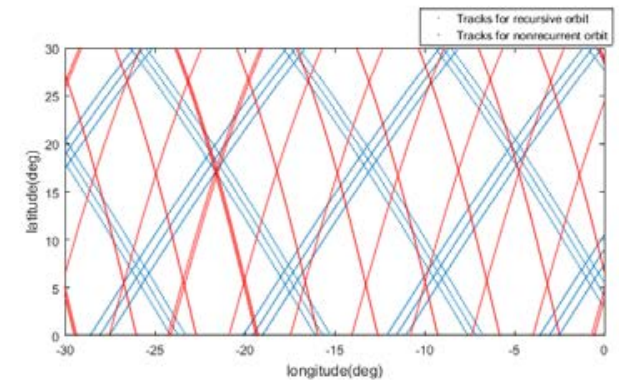

FIGURE.2 The target area and ground tracks

\section{Model for Recursive Orbit Parameters Calculation}

As the recursive orbit has great advantage in area coverage, then the model for calculation of recursive orbit should be researched. Taking no account of the orbit perturbation, the orbit period can be represented with

$$
T_{s}=\frac{2 \pi D}{L \omega_{e}}
$$

where $D$ is regression days, $L$ is regression circles and $\omega_{e}$ is angle speed of the Earth rotation. Combine with the equation

$$
T_{s}=2 \pi \sqrt{a^{3} / \mu}
$$

the orbit parameter can be carried out. But for LEO satellite, perturbation of nonspherical Earth has great influence on the ground tracks. The first order long-term items of $J_{2}$ item can be represented with [11]

$$
\dot{\Omega}=-\frac{3 \sqrt{\mu} J_{2} r_{E}^{2}}{\left(1-e^{2}\right)^{2} a^{7 / 2}} \cos i
$$


and

$$
\dot{\omega}=\dot{\Omega} \frac{(5 / 2) \sin ^{2} i-2}{\cos i}
$$

Then the orbit period is

$$
T_{s}=2 \pi\left(\frac{a^{3}}{\mu}\right)^{1 / 2}\left[1-\frac{3 J_{2} r_{E}^{2}}{2 a^{2}}\left(2-\frac{5}{2} \sin ^{2} i\right)\right]
$$

Then the equation (1) should be corrected as

$$
T_{s}=\frac{2 \pi D}{L\left(\omega_{e}-\dot{\Omega}\right)}
$$

In orbit design, we usually can only get the altitude range $h_{\max }$ and $h_{\min }$ from the resolution requirement and $D$ and $L$ from reconnaissance requirement. The other parameters are difficult to be calculated from above transcendental equations. Here the iteration algorithm is researched, as Fig.3 shows.

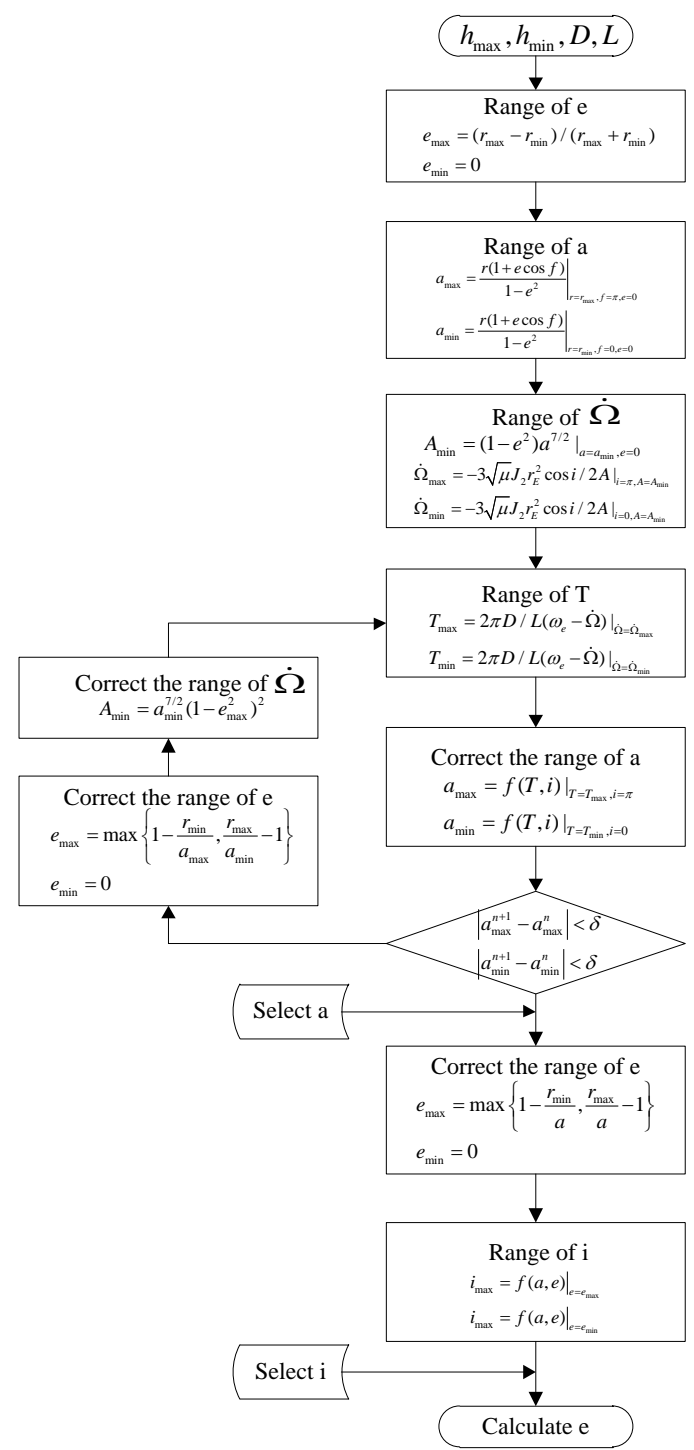

FIGURE.3 Iteration algorithm for recursive orbit parameters

\section{Configuration for LEO Satellite Constellation}

In the research of modern evolutionary algorithm for satellite constellation, the Walker type is always selected to be the basic configuration as it has good performance in whole earth coverage $[12,13]$. But for area discontinuous coverage, configurations with common ground tracks always have better performances than that of Walker types. To design a good performance for area 
coverage based on the criterions above, the advantages of recursive orbit and common ground tracks are combined and the recursive configuration with common ground tracks is researched.

For photo-reconnaissance satellites, orbit altitude and optic angle are always limited by the requirement of ground resolution [14]. Then the coverage circle radius is less than longitude spacing between two adjacent tracks, leading to incomplete coverage of target area, as figure 4 shows. So in order to enlarge the coverage area, enough tracks to be designed in the gaps.

Here define all the ground tracks of one satellite in recursive orbit as one strip of ground track. Then similar with the Walker type, the new configuration can be identified with three parameters: $N / P / F$, where $N$ is the total amount of satellites, $P$ is the amount of strips of tracks, and $F$ is the phase factor between two satellite in adjacent strip of ground track. Then the amount of satellites in one strip of ground track is

$$
S=\frac{N}{P}
$$

Then the phase spacing between two adjacent satellites in one strip of track is

$$
\Delta u=\frac{2 \pi L}{S}
$$

where $L$ is the regression circles Phase spacing between two adjacent ascending nodes in different strip of ground tracks is

$$
\Delta \lambda=\frac{2 \pi}{P L}
$$

Then the phase spacing between adjacent satellites in adjacent strips of tracks is

$$
\delta u=F \frac{2 \pi L}{N}
$$

Then for the $j$ th satellite in the $i$ th strip of track ( $s_{i, j}$ for short), the phase should be

$$
u_{i, j}=u_{0,0}+(i-1) \delta u+(j-1) \Delta u
$$

where $u_{0,0}$ is the phase of the first satellite. Then the difference time of ascending nodes between $s_{i, j}$ and $s_{0,0}$ is

$$
d t=\frac{M_{0,0}-M_{i, j}}{n}
$$

where $M$ is the anomaly and $n$ is the mean motion. Then the perturbation of the $J_{2}$ item can be calculated by

$$
\varepsilon \Omega=\left(\dot{\Omega}-\omega_{e}\right) d t
$$

Then the RAAN of $s_{i, j}$ is

$$
\Omega_{i, j}=\Omega_{0,0}+(i-1) \Delta \lambda+\varepsilon \Omega
$$

\section{Simulations}

To verify the advantage of the new configuration, a comparison of coverage performance between Walker type and the recursive configuration with common ground tracks. The two configuration have the same parameters $N / P / F=12 / 3 / 1$. The common orbit parameters are $a=7365.67 \mathrm{~km}, e=7.55 \mathrm{e}-02, \Omega_{0,0}=0^{\circ}, \omega=0^{\circ}, u_{0,0}=0^{\circ}$. The inclinations are $79.06^{\circ}$ for Walker type and $100.94^{\circ}$ for the new configuration. The optic angle of the sensors is $9.0^{\circ}$. As the regression period is $D=4 d$ and regression circle is $L=55$, then the simulation time is selected to be 12 days. Then the ground tracks of these two configurations are shown in Fig.4 and Fig.5. 


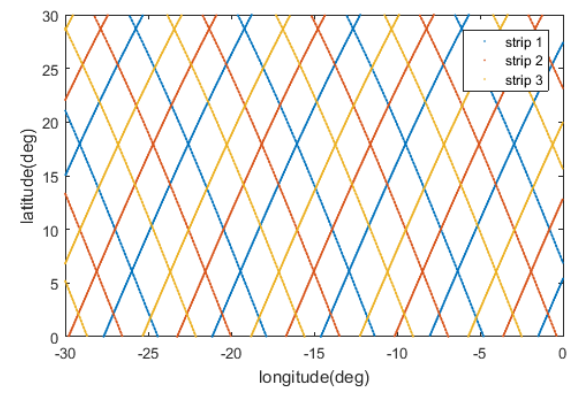

FIGURE.4 Ground track of new configuration

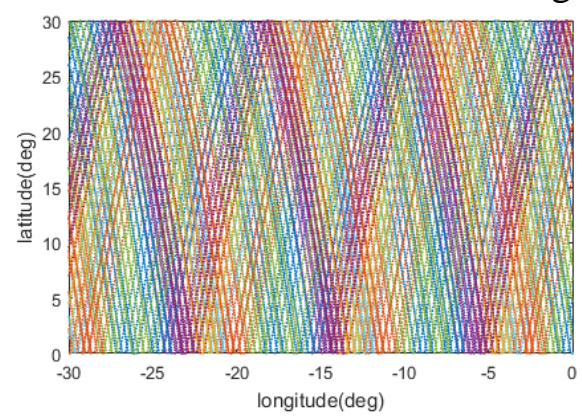

FIGURE.5 Ground track of Walker type

Select the target area with the longitude range of $\left(30^{\circ} \mathrm{W}, 0^{\circ}\right)$ and latitude range of $\left(0^{\circ}, 30^{\circ} \mathrm{N}\right)$. As the common coverage performance indexes were designed for point target, two new indexes are designed for evaluate the coverage performance for area target. One index is the mean coverage time $T_{m c}$, which is defined as the mean value of coverage time of all the unit area. The other one is the mean coverage gap $T_{m g}$, which is defined as the sum value of coverage gap of all the unit area divided to whole area and each gap number. Using $c_{i}, g_{i}$ and $n_{i}$ as identifications of coverage time, coverage gap and gap number of the $i$ th unit area, then the two indexes can be represented as

$$
T_{m c}=\sum c_{i} / A_{\text {total }}
$$

and

$$
T_{m g}=\frac{1}{A_{\text {total }}} \sum \frac{g_{i}}{n_{i}}
$$

where $A_{\text {total }}$ is the total size of target area. Then the coverage performances of the two configurations are shown in Fig.6, Fig.7 and Table 1.

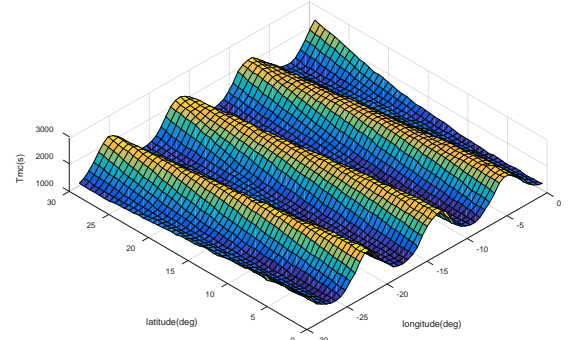

(a)

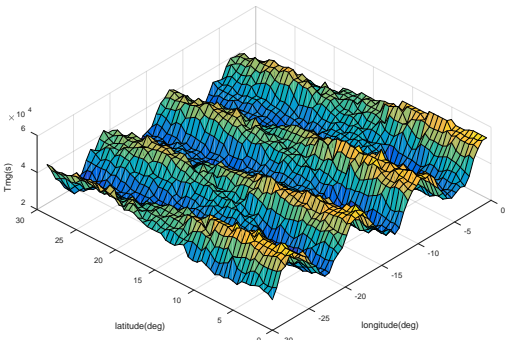

(b)

FIGURE.6 Coverage performance for Walker type 


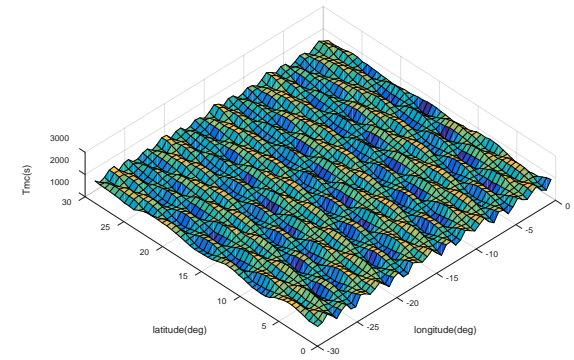

(a)

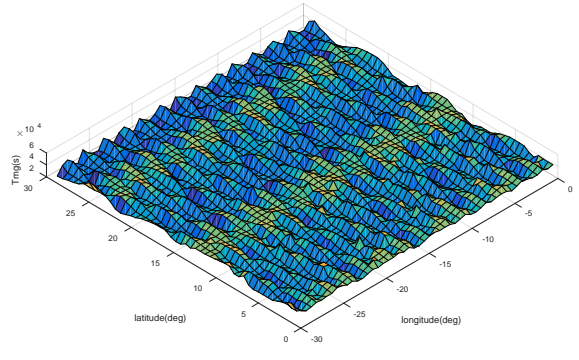

(b)

FIGURE.7 Coverage performance for new configuration

The result in Fig.6 and Fig.7 suggests that the new configuration have more even distribution of the mean coverage time and mean coverage gap than that of Walker type. It's more suitable for reconnaissance satellite constellation, because the even distribution of $T_{m c}$ and $T_{m g}$ means the less detection time delay of time-sensitive stochastic target. The data in table.1 suggests that the new configuration have same $T_{m c}$ but less $T_{m g}$ indexes than Walker type.

Table.1 Coverage performance comparison

\begin{tabular}{ccc}
\hline $\begin{array}{c}\text { Configuration } \\
\text { type }\end{array}$ & $T_{m c} / \mathrm{s}$ & $T_{m g} / \mathrm{s}$ \\
\hline $\begin{array}{c}\text { Walker } \\
\text { configuration }\end{array}$ & 1800.4 & 32209.7 \\
New configuration & 1795.6 & 31196.3 \\
\hline
\end{tabular}

\section{Conclusion}

To design configuration suit for area coverage, the orbit selection criterions are put forward. The criterion suggested that the recursive orbit and configuration with common tracks are more suit for area coverage. Then the iterative algorithm taking account of the perturbation of $J_{2}$ item is researched. In addition to this, the advantages are combined and the new configuration is designed further. In the final simulation, the coverage performances of the new configuration and Walker configuration are compared. The result suggested that the new configuration have better coverage performance than Walker type and is more suit for area coverage.

\section{Reference}

[1] Song Zhiming, Dai Guangming, Wang Maocai, etc. Continuous coverage analysis of satellite constellation to ground target[J]. J. Huazhong Univ. of Sci. \& Tech. 2014, 42(8): 33-37

[2] HU Songjie, SHEN Jingsong, HUAN Pei. A Relative Phase-Keeping Strategy of Walker Constellation Based on Reference Orbit[J]. Aerospace Control and Application, 2010, 36(5): 45-49

[3] WEI Juan, ZHANG Run. Optimization design and simulation analysis of earth reconnaissance satellite constellation[J]. Journal of Xidian University, 2013, 40(2): 138-147

[4] CHENG Siwei, ZHANG Hui, SHEN Lincheng, etc. Optimization of Regional Coverage Reconnaissance Satellite Constellation by GDE3 Algorithm[J]. 2009, Journal of System Simulation, 21(2): 586-589

[5] MENG Bo, HAN Chao. Optimization of Hybrid Constellation by Modified Particle Swarm Algorithm[J]. Aerospace Shanghai. 2010, 1: 36-39

[6] Li Sudan, ZHU Jiang, Li Guangxia. Optimization of LEO regional communication satellite constellation with GA algorithm[J]. Journal on Communication. 2005, 26(8): 122-128

[7] WANG Li, WANG Yanjuan, ZHANG Hui, etc. Optimization of Regional Coverage 
Reconnaissance Satellite Constellations by NSGA-II Algorithm[J]. Computer Simulation, 2009, 26(4): 80-88

[8] Huameng Gao, He Liu. Design of Regional Coverage Resource Satellite Constellation Based on Analytical Method[J]. Advanced Materials Research, 2014, 1049-1050: 1894-1897

[9] Huang He, Zhou Jun, Liu Yingying. Optimal Design for Multi-Task Satellite Cluster Adaptive Orbit[J]. 2014, 32(5): 834-839

[10]Guo Chao, Deng Li, Hu Yasi. Constellation Design for Regional Coverage Satellite[J]. Microcomputer Application. 2014. 30(11): 47-48

[11]SUN Yang, XU Kai, ZHANG Jing, etc. The Design and Target Coverage Simulation of Small Earth Observation Satellite Orbit[J]. Journal of Sichuan Ordnance, 2013, 34(7): 145-148

[12]SONG Zhiming, DAI Guangming, WANG Maocai, etc. Theoretical analysis of Walker constellation coverage to area target[J]. Computer Engineering and Design, 2014, 35(10): 3639-3644

[13]FAN Li, JIANG Chao, YANG Xuerong, etc. Orbital Design for On-orbit Walker Constellation Satellites Servicing[J]. Journal of Academy of Equipment, 2013, 24(1): 69-72

[14]JING Wenbo, GAO Yongming, WU Zhihuan, etc. Multi-software Co-Optimization of Minisat Orbit for Observation Mission[J]. Computer Simulation, 2015, 32(2): 43-47 